\title{
Simulation Study of the Observed Radio Emission of Air Showers by the IceTop Surface Extension
}

\section{The IceCube Collaboration}

(a complete list of authors can be found at the end of the proceedings)

E-mail: alanc@udel.edu

Multi-detector observations of individual air showers are critical to make significant progress to precisely determine cosmic-ray quantities such as mass and energy of individual events and thus bring us a step forward in answering the open questions in cosmic-ray physics. An enhancement of IceTop, the surface array of the IceCube Neutrino Observatory, is currently underway and includes adding antennas and scintillators to the existing array of ice-Cherenkov tanks. The radio component will improve the characterization of the primary particles by providing an estimation of $X_{\max }$ and a direct sampling of the electromagnetic cascade, both important for per-event mass classification. A prototype station has been operated at the South Pole and has observed showers, simultaneously, with the tanks, scintillator panels, and antennas. The observed radio signals of these events are unique as they are measured in the 70 to $350 \mathrm{MHz}$ band, higher than many other cosmic-ray experiments. We present a comparison of the detected events with the waveforms from CoREAS simulations, convoluted with the end-to-end electronics response, as a verification of the analysis chain. Using the detector response and the measurements of the prototype station as input, we update a Monte-Carlo-based study on the potential of the enhanced surface array for the hybrid detection of air showers by scintillators and radio antennas.

Corresponding author: Alan Coleman ${ }^{1 *}$

${ }^{1}$ Bartol Research Institute and University of Delaware Department of Physics \& Astronomy, Newark, DE, USA

* Presenter

$37^{\text {th }}$ International Cosmic Ray Conference (ICRC 2021)

July 12 th $-23 r d, 2021$

Online - Berlin, Germany 


\section{CR Detection Using Surface-Radio at the South Pole}

For the next generation of cosmic ray (CR) arrays, a more accurate identification of the properties of the primary particle is a driving concern for the design of detector components. In the field of high-energy CRs, there are still correlated unknowns such as the origins and acceleration mechanisms. A major challenge to making progress towards these open questions is the underlying $\mathrm{CR}$ mass distributions. The second unknown is an accurate description of the hadronic interactions that govern particle generation at center-of-mass energies above those measured at the LHC. Current experiments have shown that above $\mathrm{CR}$ energies of $\sim 30 \mathrm{PeV}$, modern hadronic interaction models do not accurately reproduce the observed distribution of muons [1]. One method towards making progress in understanding these linked topics is to disentangle the distributions of air shower particles, specifically the electromagnetic (EM) and muonic content.

Radio antennas have reached maturity in their use to detect air showers and are only sensitive to the EM content of the shower. The ability to determine the EM energy and $\mathrm{X}_{\max }$ has already been demonstrated by experiments such as the Pierre Auger Observatory [2], LOFAR [3], and Tunka-Rex [4] to an accuracy of better than $20 \%$ and $30 \mathrm{~g} \mathrm{~cm}^{-2}$, respectively. These quantities are important when trying to classify the primary mass of a given air shower observation.

IceTop is a $1 \mathrm{~km}^{2} \mathrm{CR}$ detector and is part of the IceCube Neutrino Observatory, located at the South Pole. It currently consists of 162 ice Cherenkov tanks which detect the emission of relativistic particles that enter their volume. The IceTop enhancement foresees the addition of scintillator panels and radio antennas to the current footprint to extend the CR and neutrino program of the Observatory [5]. In this work, we detail the end-to-end simulation chain of the antennas in section 2, present a calculation of the expected sensitivity of the final array in section 3.2, and show a comparison of simulated and observed waveforms using the currently deployed prototype station $[6,7]$ in section 3.3.

\section{Simulation and Analysis Software}

The suite of analysis software for the processing of observed and simulated radio waveforms is included in the larger IceCube framework, IceTray [8]. The additional radio-specific software includes a repository of modules which are used to analyze waveforms in the time and frequency domains, (de)convolve the hardware responses, perform frequency filtering (see section 3.1), and calculate standard physics quantities such as the radio-frequency energy fluence.

For the results shown in this work, the air-shower Monte Carlo (MC) package, CORSIKA, and its radio emission extension, CoREAS, were used to calculate both the particle emission and electric field waveforms [9] for the proposed IceTop Enhancement layout.

The injection of the CR air-shower secondaries into the particle detectors, the resulting light yield, and the photo-multiplier response are handled with a dedicated Geant 4 simulation on a perevent basis [10]. The fidelity of the scintillator injection code is particularly important as the panels provide the external trigger for the readout of the antennas and are therefore an integral part of simulating air shower events with antenna waveforms.

The simulation of the radio response begins with the output of the CoREAS package, the electric field as a function of time, $\vec{E}(t)$. The beginning of the waveform is first pre-padded with 


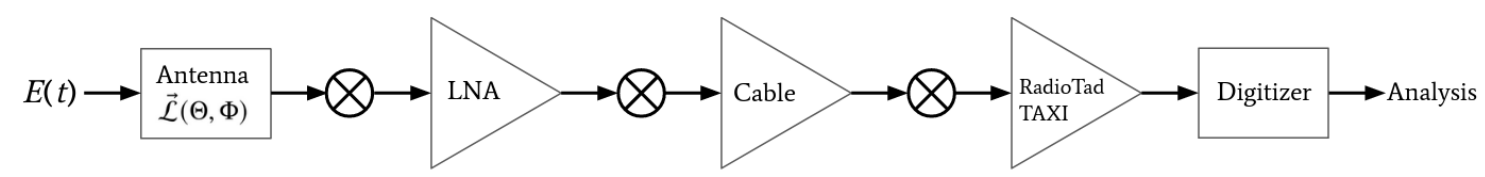

Figure 1: A schematic of the simulation chain is shown above. The voltage in the antenna is calculated using a simulated model of the vector effective length. Convolutions are used to account for the various hardware components after which there is a final digitization to ADC units.

$\overrightarrow{0}$ such that it is 5000 bins long and then resampled from the original $0.2 \mathrm{~ns}$ time-step binning to $1 \mathrm{~ns}$, producing a waveform that is $1 \mu \mathrm{s}$ long. The far-field antenna response of the SKALA v2 crossed-dipole was simulated for all Poynting vector directions in steps of $1^{\circ}$ and in frequencies in $1 \mathrm{MHz}$ steps for 50 - $350 \mathrm{MHz}$ [11]. The simulated vector effective length, $\overrightarrow{\mathcal{L}}(\Theta, \Phi)$, where $\Theta$ and $\Phi$ define the propagation direction of the far-field wave front in spherical coordinates, is used to calculate the voltage in the antenna, $V(f)=\overrightarrow{\mathcal{L}}(\Theta, \Phi ; f) \cdot \vec{E}(f)$.

The voltages that are produced in the SKALA antenna are then folded with the response of the hardware readout chain, including a low-noise amplifier (LNA), coaxial cables, and a pre-processing board. All components are assumed to be working in their linear regime and thus can be directly combined as shown in fig. 1. The gain of the LNA, mounted to the top of each SKALA antenna, has been simulated and provides an approximate $+40 \mathrm{~dB}$ amplification. Next, the $50 \mathrm{~m}$ LMR400 coaxial cables, the response of which has been measured as a function frequency and temperature, is -2 to $-1 \mathrm{~dB}$, with higher attenuation at high frequency. Finally the voltage is folded with the DAQ which includes the signal pre-processing

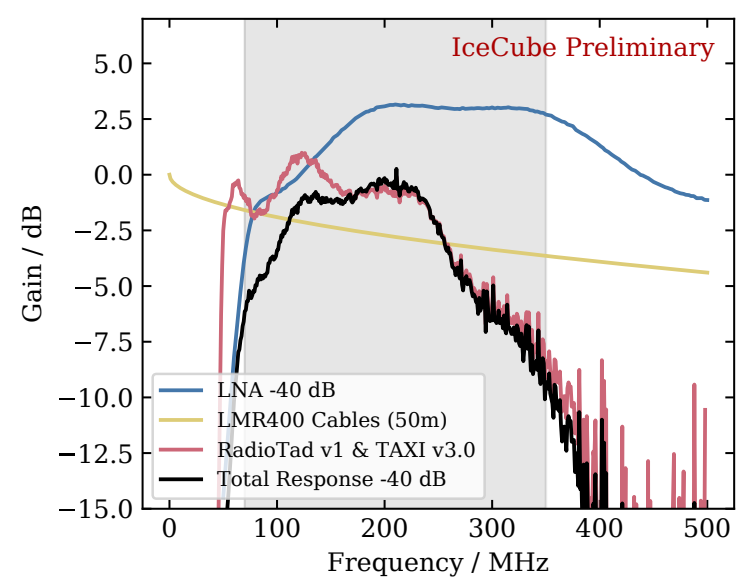

Figure 2: The power gain of the various components is shown above. The gray band indicates the nominal frequency range of the surface array, $70-350 \mathrm{MHz}$. board, RadioTad, and an ADC converter, TAXI v3.0 [6]. The combined response of the DAQ has been measured in the lab and is -10 to $+1 \mathrm{~dB}$, varying over the frequency band of interest. The frequency-dependent responses of the components described above are shown in fig. 2. The final step includes a digitization of the signal with 14 bit precision and a dynamic range of $1 \mathrm{~V}$. The combined response of this signal chain ensures no saturation for CR energies up to $10^{18} \mathrm{eV}$.

\section{Detection of CR Events Using Surface Radio}

The sensitivity of the antenna array to air showers is determined by the density of the antennas, the external trigger of the scintillator panels, and the characteristics of the background noise. The array will include 92 antennas distributed over the current IceTop footprint. The primary background is a result of the diffuse flux emitted from Galactic and extra-Galactic sources with additional spikes caused by terrestrial radio-frequency interference (RFI). This section will discuss 


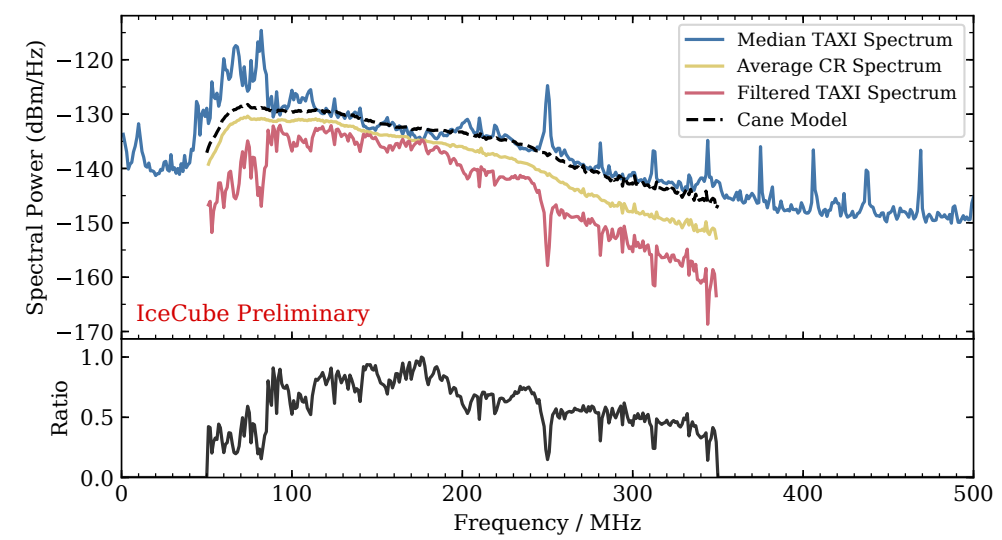

Figure 3: The median spectral power of the local background as measured by the prototype station at the South Pole using TAXI v3.0 [6] is shown in blue. The Cane model of Galactic and extra-Galactic diffuse radio emission, folded with the detector response, is shown in dashed black. The average CR emission spectrum and the resultant frequency weighting are shown in gold and red, respectively. The bottom plot directly shows the frequency weights, eq. (1), directly.

a method to weight the individual signal frequencies, a calculation of the sensitivity of the antenna array, and a comparison of observed and simulated waveforms.

\subsection{Post-processing Frequency Filters}

The spectral power from background noise has been characterized using measurements from the prototype station at the South Pole deployed in 2020, which includes the radioTad v1 and TAXI v3.0 readout system [6] and is shown in fig. 3 (blue). The distribution is the bin-wise median of 10k $1 \mu \mathrm{s}$ waveforms. For comparison, the Cane model [12] of Galactic and extra-Galactic diffuse radio emission has been folded with the antenna and detector responses as described in section 2 (dashed black). Above $100 \mathrm{MHz}$, the observed power is consistent with the Cane model with additional RFI peaks (ex: at $250 \mathrm{MHz}$ ). Below $100 \mathrm{MHz}$, the prototype version of TAXI emits power which is $10 \mathrm{~dB}$ above the Cane model. This is generated by the DC-DC converters and has been mitigated in the improved version (TAXI v3.2) that will be deployed in the future Pole seasons [6]. Other RFI peaks are evident at the highest energies but are above the nominal band where detection is limited by $-150 \mathrm{dBm} / \mathrm{Hz}$ thermal noise.

Standard methods exist for reducing coherent noise such as applying a median filter to individually measured waveforms, wherein the amplitudes of individual frequency bins, $a_{i}$, are replaced with the median value in a window of half-width, $h w, a_{i}^{\prime}=\operatorname{Median}\left(a_{i-h w}, \ldots, a_{i+h w}\right)$. Another typical method involves applying hardware- or software-based notch filters wherein specific, narrow frequency bands are removed. We build on these two ideas to create a frequency weighting scheme which notches the signals by using the median background power spectrum without the need to choose which frequencies to notch.

For this work, a library of simulated air showers was created which included discrete zenith, azimuth, and energy bins. The directions were chosen based on the direction of the local geomagnetic field which is $17.8^{\circ}$ from the zenith and $30.7^{\circ}$ west-of-north at the South Pole. Zenith angles were chosen in $17^{\circ}$ steps from $0^{\circ}$ to $68^{\circ}$ and azimuth angles were chosen to maximize and minimize the angle with the geomagnetic field, $30.7^{\circ}$ west-of-north and east-of-south. Proton and 
iron primaries were taken as limiting cases, using Sibyll 2.3d as a hadronic-interaction model [13]. The April South Pole atmosphere was used. The radio emission at each antenna was propagated through the simulation chain described in section 2.

To construct the frequency weights, we begin the with bin-wise median amplitudes, $m_{i}$, of the measured background as shown in blue in fig. 3. We then calculated the average emission from $300 \mathrm{PeV}$ air showers as a function of frequency, $C_{i}$, using only the three antennas per event with the largest Hilbert peak. Finally the weighting scheme is given by the ratio of these two quantities,

$$
w_{i}=\mathcal{N}\left(C_{i} / m_{i}\right)^{p} .
$$

The parameter, $p$, can be set by the user to increase or decrease the weighting. Note that for $p=1$, the application of the weights, $w_{i}$, will simply change $a_{i} \longrightarrow C_{i}$, on average, and that for this paper, $p=2$, has been used. A normalization factor, $\mathcal{N}$, is used such that the maximum value of $w_{i}$ is 1 . The distributions of $C_{i}$ and $m_{i} \times w_{i}$ are shown in gold and red, respectively in fig. 3. The bottom plot shows the ratio from which the weights were constructed (black).

This weighting scheme, $w_{i}$, goes beyond a simple filtering and additionally accentuates frequencies where the typical air shower spectral shape is harder than that of the background, $\simeq 100$ to $200 \mathrm{MHz}$. This process also inherently applies a notch filtering where the prominence of each RFI spike becomes an equally deep notch.

\subsection{Expected Observed Events at the Pole}

The readout of the antenna waveforms will be initiated by the scintillator panels and thus all events will have corresponding waveforms. The decision to include radio information in a given antenna during an analysis relies on the ability to determine meaningful properties of the air shower pulse amongst all sources of background noise. For this, we use a basic criteria of a signal-to-noiseratio (SNR) threshold cut as defined by the square of the ratio of the peak amplitude of the Hilbert envelope and the RMS of the voltage of the noise,

$$
\mathrm{SNR}=\left(\frac{\text { HilbertPeak }}{\text { RMS }}\right)^{2}
$$

To reduce contamination of the true signal when calculating the RMS, we use separate signal and noise windows, $200 \mathrm{~ns}$ and $400 \mathrm{~ns}$ long, respectively. The size of the signal window is motivated by the $\leq 1^{\circ}$ angular resolution of the scintillator reconstruction [10] and the $\leq 300 \mathrm{~m}$ radius of the Cherenkov ring for trajectories with $\theta<65^{\circ}$.

The SNR threshold was then determined by using background waveforms measured at the Pole. Again 10k waveforms were analyzed using randomly positioned signal and noise windows with the constraint that the noise window is at least $50 \mathrm{~ns}$ after the signal window. The frequency weights, eq. (1) were applied to the waveforms and the SNR values were calculated. Since the optional frequency band to use for determining detection is a priori unknown, a final bandpass filter was applied for various high-pass (50 to $250 \mathrm{MHz}$ ) and low-pass (150 to $350 \mathrm{MHz}$ ) values. The SNR cut values which exclude $99 \%$ of background waveforms are shown in fig. 4 as a function of the considered band.

The optimal frequency band to distinguish background from CR pulses was then determined using the library of showers, described above. First, showers with energies of $300 \mathrm{PeV}$ were 

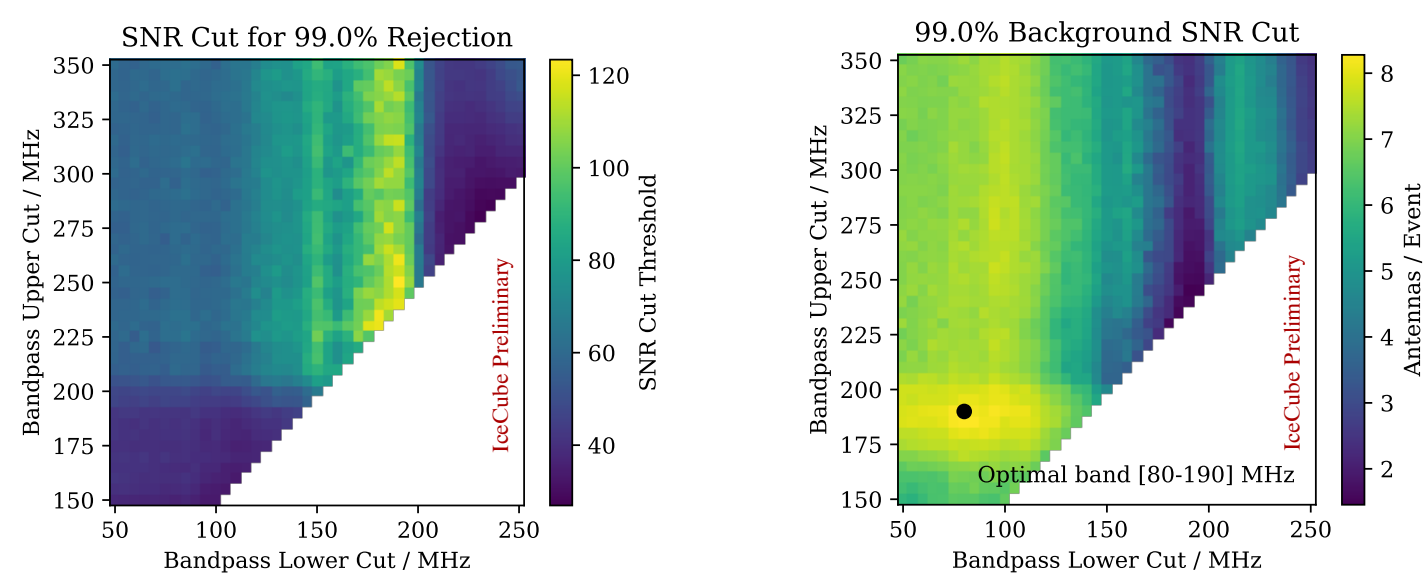

Figure 4: Left: The SNR cut values required to reject $99 \%$ of background waveforms as a function of the low- and high-pass frequencies. Right: The number of antennas per event which pass the SNR cut for the bandpass limits. The Black dot indicates the optimal band (corresponding to a SNR threshold of 41.4).

processed through the simulation chain. Background waveforms were added to the simulations directly and the frequency weights were applied. The signal window was centered on the location of the underlying peak, known from the scintillator reconstruction. The range of possible bandpasses as shown on the left in fig. 4 was applied and the measured SNR value was checked against the corresponding threshold. The optimal band was then taken to be that which maximized the average number of antennas per event that passed the SNR cut. The detection efficiency (see below) was calculated and the above procedure was again repeated but instead using all the direction and energy bins for which the array has a $30 \%$ to $70 \%$ chance to trigger. The optimal band was found to be 80 to $190 \mathrm{MHz}$, as shown on the right in fig. 4, a band consistent to a previous study which used only ideal noise [14].

Using the library of air showers, the reconstruction efficiency was determined using the full simulation chain including the addition of background noise waveforms, frequency-weighting scheme, and optimal band, as defined above. Further, the scintillator trigger algorithm was applied wherein stations were individually triggered with the requirement that at least 3 stations recorded $\geq 0.5$ MIP (minimum-ionizing particle). Using the antennas in the triggered stations which also passed the SNR cut, a plane front reconstruction was performed. If the direction of the plane wave and the MC truth agreed to within $5^{\circ}$, the event was considered successfully reconstructed. The reconstruction efficiency for proton and iron primaries is shown in fig. 5 as a function of energy and zenith angle for the best- and worst-case azimuth angles. The reconstruction efficiency for proton is better for high angles, consistent with the increased EM content compared to heavier primaries. However, for more vertical trajectories, a better efficiency is seen for the iron primaries. The yearly average atmospheric overburden at the South Pole is about $690 \mathrm{~g} / \mathrm{cm}^{2}$ which is approximately the $\left\langle X_{\max }\right\rangle$ of a vertical $100 \mathrm{PeV}$ proton shower. Thus, for more vertical protons at-and-above this energy, much of the radio emission is being truncated by the ground and not radiated to the antennas. Additionally, the $\simeq 1^{\circ}$ Cherenkov opening angle is not wide enough to produce a distinct ring on the ground and thus the footprint is thus relatively small for zenith angles less than $40^{\circ}$. For $68^{\circ}$ showers, the reconstruction efficiency is limited by the sensitivity of the scintillators to provide an external trigger. 

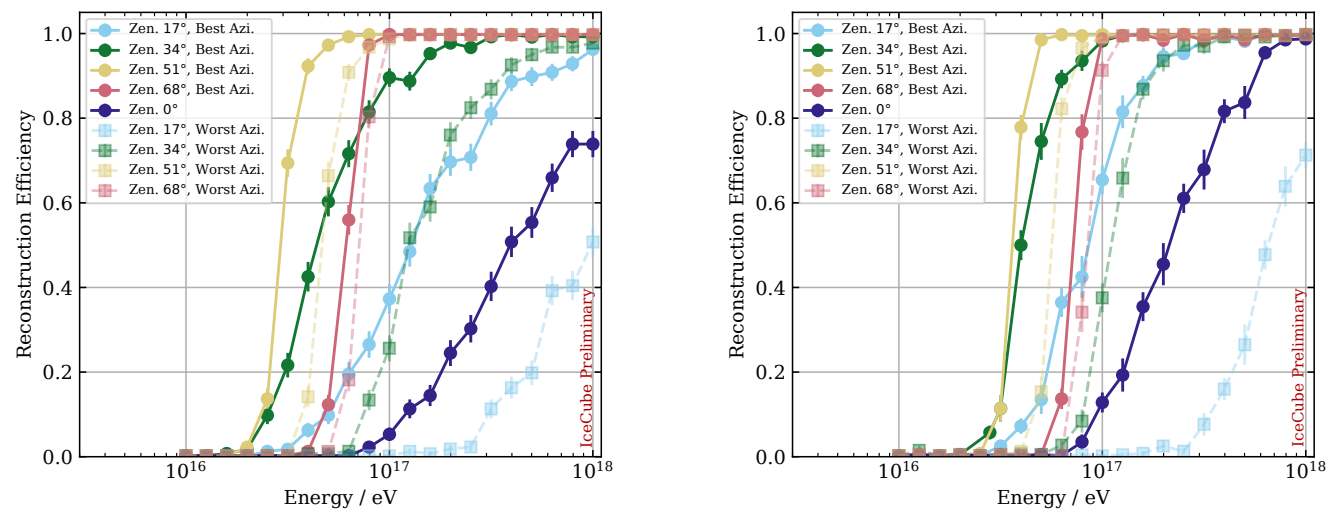

Figure 5: The fraction of simulated CoREAS events which can be reconstructed using a plane wave to within $5^{\circ}$ of the MC truth are shown for proton (left) and iron (right) primaries. Background waveforms from the South Pole, recorded with TAXI v3.0 DAQ, were used. The selection of antennas to use in the reconstruction includes an SNR cut that rejects $99 \%$ of background pulses.

\subsection{Comparison to Observed Events}

A number of events have already been observed using the prototype station at the Pole. The timestamp of events that were triggered by the scintillator array have been cross checked with the IceTop tanks as a confirmation that the array is triggering on air showers [7]. Both the IceTop and scintillator detectors are sensitive to air showers with energies at and below $1 \mathrm{PeV}$ and will thus provide simultaneous estimates of the air-shower properties. Above $30 \mathrm{PeV}$, the IceTop and scintillator reconstruction accuracy is $\sigma_{\text {core }} \leq 10 \mathrm{~m}, \psi_{\text {direction }} \leq 1^{\circ}$, and $\sigma_{E} / E \leq 15 \%$.

As a last cross check of the radio simulation chain, air showers consistent with the IceTop reconstruction were created. The radio emission was propagated into the three antennas of the prototype station and the frequency weighting scheme was applied. An example of the simulated and observed waveforms of a $32^{\circ}, 240 \mathrm{PeV}$ air shower is shown in fig. 6. Qualitatively, the simulated waveforms look to be consistent with that of the expected ones in phase and absolute amplitude, giving further confidence that the antennas are indeed recording air shower pulses.

Further systematic studies to determine consistency with the expected distribution of flux are ongoing [7]. So far the arrival distribution, corresponding estimated energies from the IceTop reconstruction, and core distributions show agreement.

\section{Conclusion}

The radio antennas will be an important component to the enhanced IceTop array. With the ability to determine the electromagnetic energy and the depth of shower maximum of CR air showers, future analyses will have increased mass discrimination power. In this work we detailed a method to weight individual frequencies to remove RFI spikes. Using this technique, the efficiency to reconstruct air showers using at least 3 radio antennas was determined. For the most vertical showers, $\leq 17^{\circ}$, reconstructions using the antennas are only possible above $100 \mathrm{PeV}$. For more inclined showers, up to $50^{\circ}$, the energy threshold is as low as $30 \mathrm{PeV}$, with strong azimuthal dependence. For the most inclined showers studied here, $68^{\circ}$, a reconstruction using the antennas is limited by the external scintillator trigger. 

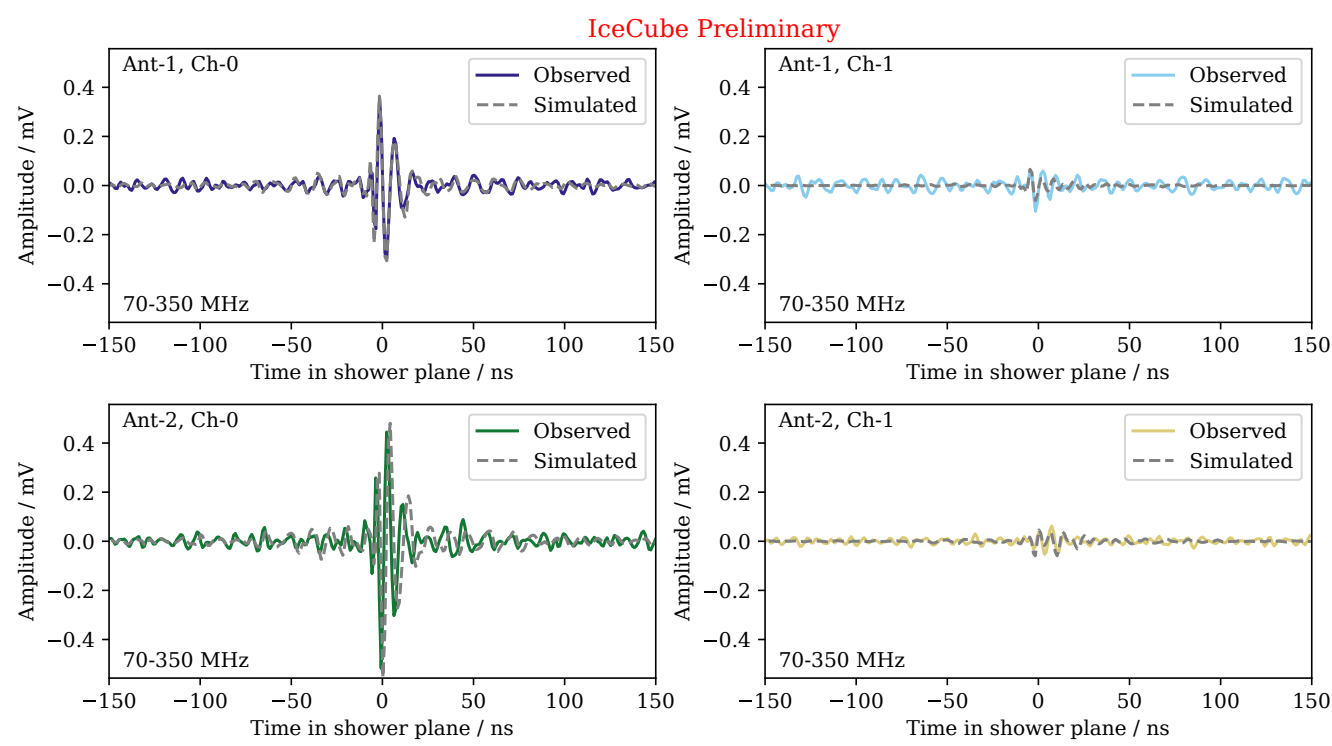

Figure 6: Voltage waveforms from an observed event (solid color) compared to the waveforms from a CoREAS simulation (dashed gray) with initial conditions as given by the IceTop reconstruction $\left(\theta=32^{\circ}\right.$, $\left.\phi=275^{\circ}, E_{\mathrm{IT}}=240 \mathrm{PeV}\right)$.

Further improvements to enhance the sensitivity of the antennas to air showers are being explored including machine learning techniques to remove noise from the waveforms directly [15, 16]. Even for the current detection thresholds, an increase in selected antennas can already provide a large boost as other experiments have shown that five or more antennas are needed to reconstruct $\mathrm{X}_{\max }$ with precision comparable to optical techniques.

Acknowledgement: U.S. National Science Foundation-EPSCoR (RII Track-2 FEC, award ID 2019597)

\section{References}

[1] D. Soldin et al. PoS ICRC2021 (2021) 349.

[2] Pierre Auger Collaboration Phys. Rev. D 93 no. 12, (2016) 122005.

[3] LOFAR Collaboration, S. Buitink et al. Phys. Rev. D 90 no. 8, (2014) 082003.

[4] Tunka-Rex Collaboration Phys. Rev. D 97 no. 12, (2018) 122004.

[5] IceCube Collaboration, F. G. Schröder PoS ICRC2019 (2019) 418.

[6] IceCube Collaboration, M. Oehler and R. Turcotte PoS ICRC2021 (2021) 225.

[7] IceCube Collaboration, H. Dujmović, A. Coleman, and M. Oehler PoS ICRC2021 (2021) 314.

[8] IceCube Collaboration, T. De Young Proceedings of CHEP 2004 (2005) 463-466.

[9] D. Heck et al. Report FZKA 6019 (1998) .

[10] IceCube Collaboration, A. Leszczyńska and M. Plum PoS ICRC2019 (2020) 332.

[11] E. de Lera Acedo et al. Proceedings of ICEAA 2015 (Sep., 2015) 839-843.

[12] H. V. Cane Mon. Not. R. Astron. Soc. 189 no. 3, (1979) 465-478.

[13] F. Riehn et al. Phys. Rev. D 102 no. 6, (2020) 063002.

[14] V. A. Balagopal and others Eur. Phys. J. C 78 no. 2, (2018) 1-14.

[15] A. Rehman, A. Coleman, and F. G. Schroeder PoS ICRC2021 (2021) 417.

[16] Bezyazeekov, P. and others MDPI Proc. L 2679 (2020) 43-50. 


\section{Full Author List: IceCube Collaboration}

R. Abbasi ${ }^{17}$, M. Ackermann ${ }^{59}$, J. Adams ${ }^{18}$, J. A. Aguilar ${ }^{12}$, M. Ahlers ${ }^{22}$, M. Ahrens ${ }^{50}$, C. Alispach ${ }^{28}$, A. A. Alves Jr. ${ }^{31}$, N. M. $\mathrm{Amin}^{42}$, R. An ${ }^{14}$, K. Andeen ${ }^{40}$, T. Anderson ${ }^{56}$, G. Anton ${ }^{26}$, C. Argüelles ${ }^{14}$, Y. Ashida ${ }^{38}$, S. Axani ${ }^{15}$, X. Bai ${ }^{46}$, A. Balagopal V. ${ }^{38}$, A. Barbano ${ }^{28}$, S. W. Barwick ${ }^{30}$, B. Bastian ${ }^{59}$, V. Basu ${ }^{38}$, S. Baur ${ }^{12}$, R. Bay ${ }^{8}$, J. J. Beatty ${ }^{20,21}$, K.-H. Becker ${ }^{58}$, J. Becker Tjus ${ }^{11}$, C. Bellenghi $^{27}$, S. BenZvi ${ }^{48}$, D. Berley ${ }^{19}$, E. Bernardini ${ }^{59}, 60$, D. Z. Besson ${ }^{34,61}$, G. Binder ${ }^{8}, 9$, D. Bindig ${ }^{58}$, E. Blaufuss ${ }^{19}$, S. Blot ${ }^{59}$, M. Boddenberg ${ }^{1}$, F. Bontempo ${ }^{31}$, J. Borowka ${ }^{1}$, S. Böser ${ }^{39}$, O. Botner ${ }^{57}$, J. Böttcher ${ }^{1}$, E. Bourbeau ${ }^{22}$, F. Bradascio ${ }^{59}$, J. Braun ${ }^{38}$, S. Bron $^{28}$, J. Brostean-Kaiser ${ }^{59}$, S. Browne ${ }^{32}$, A. Burgman ${ }^{57}$, R. T. Burley ${ }^{2}$, R. S. Busse ${ }^{41}$, M. A. Campana ${ }^{45}$, E. G. Carnie-Bronca ${ }^{2}$, C. Chen ${ }^{6}$, D. Chirkin ${ }^{38}$, K. Choi ${ }^{52}$, B. A. Clark ${ }^{24}$, K. Clark ${ }^{33}$, L. Classen ${ }^{41}$, A. Colemann ${ }^{42}$, G. H. Collin ${ }^{15}$, J. M. Conrad ${ }^{15}$, P. Coppin $^{13}$, P. Correa ${ }^{13}$, D. F. Cowen ${ }^{55,56}$, R. Cross $^{48}$, C. Dappen ${ }^{1}$, P. Dave ${ }^{6}$, C. De Clercq ${ }^{13}$, J. J. DeLaunay ${ }^{56}$, H. Dembinski ${ }^{42}$, K. Deoskar $^{50}$, S. De Ridder ${ }^{29}$, A. Desai ${ }^{38}$, P. Desiati ${ }^{38}$, K. D. de Vries ${ }^{13}$, G. de Wasseige ${ }^{13}$, M. de With ${ }^{10}$, T. DeYoung ${ }^{24}$, S. Dharani ${ }^{1}$, A. Diaz ${ }^{15}$, J. C. Díaz-Vélez ${ }^{38}$, M. Dittmer ${ }^{41}$, H. Dujmovic ${ }^{31}$, M. Dunkman ${ }^{56}$, M. A. DuVernois ${ }^{38}$, E. Dvorak ${ }^{46}$, T. Ehrhardt ${ }^{39}$, P. Eller $^{27}$, R. Engel $^{31,}{ }^{32}$, H. Erpenbeck ${ }^{1}$, J. Evans ${ }^{19}$, P. A. Evenson ${ }^{42}$, K. L. Fan ${ }^{19}$, A. R. Fazely ${ }^{7}$, S. Fiedlschuster ${ }^{26}$, A. T. Fienberg ${ }^{56}$, K. Filimonov ${ }^{8}$, C. Finley ${ }^{50}$, L. Fischer ${ }^{59}$, D. Fox ${ }^{55}$, A. Franckowiak ${ }^{11,59}$, E. Friedman ${ }^{19}$, A. Fritz ${ }^{39}$, P. Fürst ${ }^{1}$, T. K. Gaisser ${ }^{42}$, J. Gallagher $^{37}$, E. Ganster ${ }^{1}$, A. Garcia ${ }^{14}$, S. Garrappa ${ }^{59}$, L. Gerhardt ${ }^{9}$, A. Ghadimi ${ }^{54}$, C. Glaser ${ }^{57}$, T. Glauch ${ }^{27}$, T. Glüsenkamp ${ }^{26}$, A. Goldschmidt $^{9}$, J. G. Gonzalez ${ }^{42}$, S. Goswami ${ }^{54}$, D. Grant ${ }^{24}$, T. Grégoire ${ }^{56}$, S. Griswold ${ }^{48}$, M. Gündüz ${ }^{11}$, C. Günther ${ }^{1}$, C. Haack ${ }^{27}$,

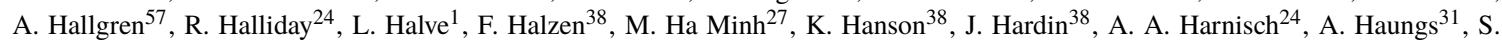

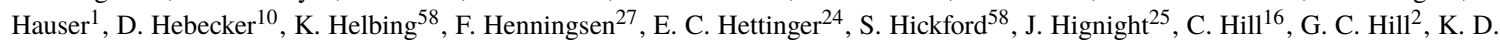

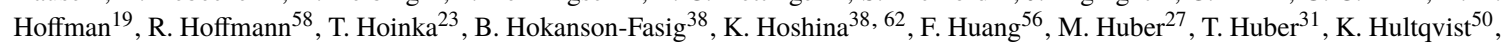
M. Hünnefeld ${ }^{23}$, R. Hussain ${ }^{38}$, S. In ${ }^{52}$, N. Iovine ${ }^{12}$, A. Ishihara ${ }^{16}$, M. Jansson ${ }^{50}$, G. S. Japaridze ${ }^{5}$, M. Jeong ${ }^{52}$, B. J. P. Jones ${ }^{4}$, D. Kang ${ }^{31}$, W. Kang ${ }^{52}$, X. Kang ${ }^{45}$, A. Kappes ${ }^{41}$, D. Kappesser ${ }^{39}$, T. Karg ${ }^{59}$, M. Karl ${ }^{27}$, A. Karle ${ }^{38}$, U. Katz ${ }^{26}$, M. Kauer ${ }^{38}$, M. Kellermann ${ }^{1}$, J. L. Kelley $^{38}$, A. Kheirandish ${ }^{56}$, K. Kin ${ }^{16}$, T. Kintscher ${ }^{59}$, J. Kiryluk ${ }^{51}$, S. R. Klein ${ }^{8,9}$, R. Koirala ${ }^{42}$, H. Kolanoski ${ }^{10}$, T. Kontrimas ${ }^{27}$, L. $\mathrm{Köpke}^{39}$, C. Kopper ${ }^{24}$, S. Kopper ${ }^{54}$, D. J. Koskinen ${ }^{22}$, P. Koundal ${ }^{31}$, M. Kovacevich ${ }^{45}$, M. Kowalski ${ }^{10}$, 59, T. Kozynets ${ }^{22}$, E. Kun ${ }^{11}$, N. Kurahashi ${ }^{45}$, N. Lad ${ }^{59}$, C. Lagunas Gualda ${ }^{59}$, J. L. Lanfranchi ${ }^{56}$, M. J. Larson ${ }^{19}$, F. Lauber ${ }^{58}$, J. P. Lazar ${ }^{14,38}$, J. W. Lee ${ }^{52}$, K. Leonard $^{38}$, A. Leszczyńska ${ }^{32}$, Y. Li ${ }^{56}$, M. Lincetto ${ }^{11}$, Q. R. Liu ${ }^{38}$, M. Liubarska ${ }^{25}$, E. Lohfink ${ }^{39}$, C. J. Lozano Mariscal ${ }^{41}$, L. Lu ${ }^{38}$,

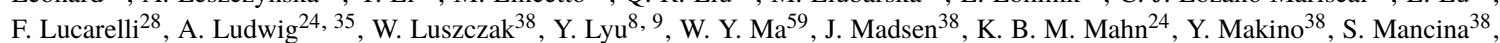

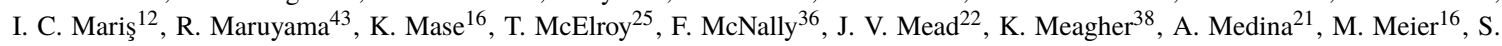
Meighen-Berger $^{27}$, J. Micallef ${ }^{24}$, D. Mockler ${ }^{12}$, T. Montaruli ${ }^{28}$, R. W. Moore ${ }^{25}$, R. Morse ${ }^{38}$, M. Moulai ${ }^{15}$, R. Naab ${ }^{59}$, R. Nagai ${ }^{16}$, U. Naumann $^{58}$, J. Necker ${ }^{59}$, L. V. Nguyễn ${ }^{24}$, H. Niederhausen ${ }^{27}$, M. U. Nisa ${ }^{24}$, S. C. Nowicki ${ }^{24}$, D. R. Nygren ${ }^{9}$, A. Obertacke Pollmann ${ }^{58}$, M. Oehler ${ }^{31}$, A. Olivas ${ }^{19}$, E. O'Sullivan ${ }^{57}$, H. Pandya ${ }^{42}$, D. V. Pankova ${ }^{56}$, N. Park ${ }^{33}$, G. K. Parker ${ }^{4}$, E. N. Paudel ${ }^{42}$, L. Paul ${ }^{40}$, C. Pérez de los $\operatorname{Heros}^{57}$, L. Peters ${ }^{1}$, J. Peterson ${ }^{38}$, S. Philippen ${ }^{1}$, D. Pieloth ${ }^{23}$, S. Pieper ${ }^{58}$, M. Pittermann ${ }^{32}$, A. Pizzuto ${ }^{38}$, M. Plum ${ }^{40}$, Y. Popovych $^{39}$, A. Porcelli ${ }^{29}$, M. Prado Rodriguez ${ }^{38}$, P. B. Price ${ }^{8}$, B. Pries ${ }^{24}$, G. T. Przybylski ${ }^{9}$, C. Raab ${ }^{12}$, A. Raissi ${ }^{18}$, M. Rameez $^{22}$, K. Rawlins $^{3}$, I. C. Rea ${ }^{27}$, A. Rehman ${ }^{42}$, P. Reichherzer ${ }^{11}$, R. Reimann ${ }^{1}$, G. Renzi ${ }^{12}$, E. Resconi ${ }^{27}$, S. Reusch ${ }^{59}$, W. Rhode ${ }^{23}$, M. Richman $^{45}$, B. Riedel ${ }^{38}$, E. J. Roberts ${ }^{2}$, S. Robertson ${ }^{8,9}$, G. Roellinghoff ${ }^{52}$, M. Rongen ${ }^{39}$, C. Rott ${ }^{49}$, 52, T. Ruhe ${ }^{23}$, D. Ryckbosch ${ }^{29}$, D. Rysewyk $\mathrm{Cantu}^{24}$, I. Safa ${ }^{14,38}$, J. Saffer ${ }^{32}$, S. E. Sanchez Herrera ${ }^{24}$, A. Sandrock ${ }^{23}$, J. Sandroos ${ }^{39}$, M. Santander ${ }^{54}$, S. Sarkar ${ }^{44}$, S. Sarkar ${ }^{25}$, K. Satalecka $^{59}$, M. Scharf ${ }^{1}$, M. Schaufel ${ }^{1}$, H. Schieler ${ }^{31}$, S. Schindler ${ }^{26}$, P. Schlunder ${ }^{23}$, T. Schmidt ${ }^{19}$, A. Schneider ${ }^{38}$, J. Schneider ${ }^{26}$, F. G. Schröder ${ }^{31,42}$, L. Schumacher ${ }^{27}$, G. Schwefer ${ }^{1}$, S. Sclafani ${ }^{45}$, D. Seckel ${ }^{42}$, S. Seunarine ${ }^{47}$, A. Sharma ${ }^{57}$, S. Shefali ${ }^{32}$, M. Silva $^{38}$, B. Skrzypek ${ }^{14}$, B. Smithers ${ }^{4}$, R. Snihur ${ }^{38}$, J. Soedingrekso ${ }^{23}$, D. Soldin ${ }^{42}$, C. Spannfellner ${ }^{27}$, G. M. Spiczak ${ }^{47}$, C. Spiering ${ }^{59,}$, ${ }^{21}$, J. Stachurska $^{59}$, M. Stamatikos ${ }^{21}$, T. Stanev ${ }^{42}$, R. Stein ${ }^{59}$, J. Stettner ${ }^{1}$, A. Steuer ${ }^{39}$, T. Stezelberger ${ }^{9}$, T. Stürwald ${ }^{58}$, T. Stuttard ${ }^{22}$, G. W. Sullivan $^{19}$, I. Taboada ${ }^{6}$, F. Tenholt ${ }^{11}$, S. Ter-Antonyan ${ }^{7}$, S. Tilav ${ }^{42}$, F. Tischbein ${ }^{1}$, K. Tollefson $^{24}$, L. Tomankova ${ }^{11}$, C. Tönnis ${ }^{53}$, S. Toscano $^{12}$, D. Tosi ${ }^{38}$, A. Trettin ${ }^{59}$, M. Tselengidou ${ }^{26}$, C. F. Tung ${ }^{6}$, A. Turcati2 ${ }^{27}$, R. Turcotte ${ }^{31}$, C. F. Turley ${ }^{56}$, J. P. Twagirayezu ${ }^{24}$, B. $\mathrm{Ty}^{38}$, M. A. Unland Elorrieta ${ }^{41}$, N. Valtonen-Mattila ${ }^{57}$, J. Vandenbroucke ${ }^{38}$, N. van Eijndhoven ${ }^{13}$, D. Vannerom ${ }^{15}$, J. van Santen ${ }^{59}, \mathrm{~S}$. Verpoest $^{29}$, M. Vraeghe ${ }^{29}$, C. Walck ${ }^{50}$, T. B. Watson ${ }^{4}$, C. Weaver ${ }^{24}$, P. Weigel ${ }^{15}$, A. Weindl ${ }^{31}$, M. J. Weiss ${ }^{56}$, J. Weldert ${ }^{39}$, C. Wendt $^{38}$, J. Werthebach ${ }^{23}$, M. Weyrauch ${ }^{32}$, N. Whitehorn ${ }^{24}, 35$, C. H. Wiebusch ${ }^{1}$, D. R. Williams ${ }^{54}$, M. Wolf ${ }^{27}$, K. Woschnagg ${ }^{8}$, G. Wrede ${ }^{26}$, J. Wulff ${ }^{11}$, X. W. Xu $u^{7}$, Y. Xu ${ }^{51}$, J. P. Yanez ${ }^{25}$, S. Yoshida ${ }^{16}$, S. Yu ${ }^{24}$, T. Yuan ${ }^{38}$, Z. Zhang ${ }^{51}$

${ }^{1}$ III. Physikalisches Institut, RWTH Aachen University, D-52056 Aachen, Germany

2 Department of Physics, University of Adelaide, Adelaide, 5005, Australia

${ }^{3}$ Dept. of Physics and Astronomy, University of Alaska Anchorage, 3211 Providence Dr., Anchorage, AK 99508, USA

${ }^{4}$ Dept. of Physics, University of Texas at Arlington, 502 Yates St., Science Hall Rm 108, Box 19059, Arlington, TX 76019, USA

5 CTSPS, Clark-Atlanta University, Atlanta, GA 30314, USA

${ }^{6}$ School of Physics and Center for Relativistic Astrophysics, Georgia Institute of Technology, Atlanta, GA 30332, USA

${ }^{7}$ Dept. of Physics, Southern University, Baton Rouge, LA 70813, USA

${ }^{8}$ Dept. of Physics, University of California, Berkeley, CA 94720, USA

${ }^{9}$ Lawrence Berkeley National Laboratory, Berkeley, CA 94720, USA

${ }^{10}$ Institut für Physik, Humboldt-Universität zu Berlin, D-12489 Berlin, Germany

${ }^{11}$ Fakultät für Physik \& Astronomie, Ruhr-Universität Bochum, D-44780 Bochum, Germany

12 Université Libre de Bruxelles, Science Faculty CP230, B-1050 Brussels, Belgium

13 Vrije Universiteit Brussel (VUB), Dienst ELEM, B-1050 Brussels, Belgium

14 Department of Physics and Laboratory for Particle Physics and Cosmology, Harvard University, Cambridge, MA 02138, USA

15 Dept. of Physics, Massachusetts Institute of Technology, Cambridge, MA 02139, USA 
${ }^{16}$ Dept. of Physics and Institute for Global Prominent Research, Chiba University, Chiba 263-8522, Japan

${ }^{17}$ Department of Physics, Loyola University Chicago, Chicago, IL 60660, USA

${ }^{18}$ Dept. of Physics and Astronomy, University of Canterbury, Private Bag 4800, Christchurch, New Zealand

${ }^{19}$ Dept. of Physics, University of Maryland, College Park, MD 20742, USA

${ }^{20}$ Dept. of Astronomy, Ohio State University, Columbus, OH 43210, USA

${ }^{21}$ Dept. of Physics and Center for Cosmology and Astro-Particle Physics, Ohio State University, Columbus, OH 43210, USA

${ }^{22}$ Niels Bohr Institute, University of Copenhagen, DK-2100 Copenhagen, Denmark

${ }^{23}$ Dept. of Physics, TU Dortmund University, D-44221 Dortmund, Germany

${ }^{24}$ Dept. of Physics and Astronomy, Michigan State University, East Lansing, MI 48824, USA

${ }^{25}$ Dept. of Physics, University of Alberta, Edmonton, Alberta, Canada T6G 2E1

${ }^{26}$ Erlangen Centre for Astroparticle Physics, Friedrich-Alexander-Universität Erlangen-Nürnberg, D-91058 Erlangen, Germany

${ }^{27}$ Physik-department, Technische Universität München, D-85748 Garching, Germany

${ }^{28}$ Département de physique nucléaire et corpusculaire, Université de Genève, CH-1211 Genève, Switzerland

${ }^{29}$ Dept. of Physics and Astronomy, University of Gent, B-9000 Gent, Belgium

${ }^{30}$ Dept. of Physics and Astronomy, University of California, Irvine, CA 92697, USA

${ }^{31}$ Karlsruhe Institute of Technology, Institute for Astroparticle Physics, D-76021 Karlsruhe, Germany

${ }^{32}$ Karlsruhe Institute of Technology, Institute of Experimental Particle Physics, D-76021 Karlsruhe, Germany

${ }^{33}$ Dept. of Physics, Engineering Physics, and Astronomy, Queen's University, Kingston, ON K7L 3N6, Canada

${ }^{34}$ Dept. of Physics and Astronomy, University of Kansas, Lawrence, KS 66045, USA

${ }^{35}$ Department of Physics and Astronomy, UCLA, Los Angeles, CA 90095, USA

${ }^{36}$ Department of Physics, Mercer University, Macon, GA 31207-0001, USA

${ }^{37}$ Dept. of Astronomy, University of Wisconsin-Madison, Madison, WI 53706, USA

${ }^{38}$ Dept. of Physics and Wisconsin IceCube Particle Astrophysics Center, University of Wisconsin-Madison, Madison, WI 53706, USA

${ }^{39}$ Institute of Physics, University of Mainz, Staudinger Weg 7, D-55099 Mainz, Germany

${ }^{40}$ Department of Physics, Marquette University, Milwaukee, WI, 53201, USA

${ }^{41}$ Institut für Kernphysik, Westfälische Wilhelms-Universität Münster, D-48149 Münster, Germany

42 Bartol Research Institute and Dept. of Physics and Astronomy, University of Delaware, Newark, DE 19716, USA

${ }^{43}$ Dept. of Physics, Yale University, New Haven, CT 06520, USA

44 Dept. of Physics, University of Oxford, Parks Road, Oxford OX1 3PU, UK

${ }^{45}$ Dept. of Physics, Drexel University, 3141 Chestnut Street, Philadelphia, PA 19104, USA

${ }^{46}$ Physics Department, South Dakota School of Mines and Technology, Rapid City, SD 57701, USA

${ }^{47}$ Dept. of Physics, University of Wisconsin, River Falls, WI 54022, USA

${ }^{48}$ Dept. of Physics and Astronomy, University of Rochester, Rochester, NY 14627, USA

${ }^{49}$ Department of Physics and Astronomy, University of Utah, Salt Lake City, UT 84112, USA

50 Oskar Klein Centre and Dept. of Physics, Stockholm University, SE-10691 Stockholm, Sweden

${ }^{51}$ Dept. of Physics and Astronomy, Stony Brook University, Stony Brook, NY 11794-3800, USA

52 Dept. of Physics, Sungkyunkwan University, Suwon 16419, Korea

${ }^{53}$ Institute of Basic Science, Sungkyunkwan University, Suwon 16419, Korea

${ }^{54}$ Dept. of Physics and Astronomy, University of Alabama, Tuscaloosa, AL 35487, USA

${ }^{55}$ Dept. of Astronomy and Astrophysics, Pennsylvania State University, University Park, PA 16802, USA

${ }^{56}$ Dept. of Physics, Pennsylvania State University, University Park, PA 16802, USA

${ }^{57}$ Dept. of Physics and Astronomy, Uppsala University, Box 516, S-75120 Uppsala, Sweden

${ }^{58}$ Dept. of Physics, University of Wuppertal, D-42119 Wuppertal, Germany

${ }^{59}$ DESY, D-15738 Zeuthen, Germany

${ }^{60}$ Università di Padova, I-35131 Padova, Italy

${ }^{61}$ National Research Nuclear University, Moscow Engineering Physics Institute (MEPhI), Moscow 115409, Russia

${ }^{62}$ Earthquake Research Institute, University of Tokyo, Bunkyo, Tokyo 113-0032, Japan

*E-mail: analysis@icecube.wisc.edu 